Research Paper

\title{
Fabrication of Superhydrophobic Surfaces from Femtosecond Laser Patterned Surfaces
}

\author{
JIJO EASO GEORGE, JOHN THOMAS, ASEEFHALI BANKAPUR, C SANTHOSH and SAJAN D \\ GEORGE $^{*}$ \\ Centre for Atomic and Molecular Physics, Manipal University, Manipal 576 104, India
}

(Received on 11 November 2014; Revised on 30 November 2014; Accepted on 17 January 2014)

\begin{abstract}
A femtosecond oscillator is employed to create periodic grid patterns in a commonly used polymer, poly (methyl methacrylate), PMMA. The wettability measured in terms of static water contact angle shows that the laser patterning causes an enhancement in water contact angle and the magnitude of change depends on the irradiation fluence during writing. A water droplet placed on a laser-patterned surface spreads anisotropically and exhibits different static water contact angle along different directions. Micro-Raman and Scanning Electron Microscope studies carried out on laser patterned and nonpatterned regions clearly confirm that the observed wettability change is due to physical (morphological) changes rather than chemical changes. More interestingly, the replica of the periodic laser patterned structures created on a soft polymer, polydimethylsiloxane (PDMS), via soft lithography technique exhibits superhydrophobic behaviour with a rolling-off nature for the water droplet. This is likely to find variety of applications in diverse fields ranging from passive defogging of windscreen to waterproof clothing to non-stick cookware.
\end{abstract}

Key Words: Water Contact Angle; Femtosecond Oscillator; Superhydrophobicity; PMMA

\section{Introduction}

During the last two decades, polymers have proven to be ideal candidates for many biomedical and MEMS/NEMS applications (Jeong, et al., 2013). In order to widen the potential applications of polymers, numerous researchers have endeavoured to modify their surface morphology to tailor the surface wettability properties (Nosonovsky and Bhushan, 2007). Majority of the reported approaches utilize chemicals to modify the surface properties of polymers. These techniques offer a number of advantages including achievement of superhydrophobicity, but require high maintenance and user involvement. An alternative, "green", method offering flexibility, accuracy and negligible bulk modification is that of laser surface patterning (Tang et al., 2011). The wide popularity of the laser-based techniques can be attributed to its unique advantages such as maskless patterning, high resolution, fast ablation, precise ablation threshold and nonrequirement of a special environment. Though nanosecond lasers are employed to ablate a wide variety of materials in early studies, recent studies are mainly focused on fabricating patterns using a femtosecond laser (Wang et al., 2011). The patterning using femtosecond lasers offers additional advantages of minimum heat affected zone, rapid energy deposition, and applicability to transparent as well as opaque materials (Yamaguchi et al., 2013). In this case, a non-linear mechanism is responsible for the laser-material energy coupling in the material. The material is ionized in the focal volume, and bonds are broken directly by the result of ultrafast electronphonon interactions. As a consequence, the temperature rise and thermal diffusion in the

*Author for Correspondence: E-mail: sajan.george@manipal.edu, Tel.: 0820-2925072 
surrounding material can be neglected. Although the measured water contact angle of the laser patterned surfaces are reported to increase to $120-130^{\circ}$ due to dual scale roughness created by femtosecond laser patterning, achieving superhydrophobic (water contact angle $>170^{\circ}$ ) surfaces by direct laser patterning is still challenging. In this work, we report the influence of femtosecond oscillator induced periodic patterning on wettability of a commonly used polymer, PMMA. The reason for the observed variation in water contact angle (WCA) is investigated spectroscopically (microRaman Spectroscopy) as well as by a surface analysis technique (Scanning Electron Microscope). The WCA on the replica created on PDMS by using laser patterned PMMA as a master structure is also investigated. We believe that superhydrophobic surfaces with rolling-off nature for water droplets can find widespread applications in diverse areas such as medicine, microfluidics, superhydrophobic cloth industry, wind screens etc.

\section{Materials and Methods}

\section{Laser Patterning}

Our experiments used commercially available $2 \mathrm{~mm}$ thick PMMA sheets (Schönig+Domes, Germany) that were cleaned in doubly distilled water using an ultrasonicator for 15 minutes. These samples were then mounted on a motorized XYZ stage and irradiated in air by a Ti: sapphire laser oscillator (Femtolasers, Austria) producing 50 fs long pulses of $800 \mathrm{~nm}$ light at $5.1 \mathrm{MHz}$ repetition rate. A single-shot autocorrelator, fibre optic coupled spectrometer (Ocean Optics, USA) and fast photodiode were used to monitor the pulse width, spectrum and repetition rate, respectively. The output energy $(200 \mathrm{~nJ})$ of the femtosecond laser oscillator was controlled using a half wave plate (HWP) and thin film polarizer before it impinged on the PMMA surface through a microscope objective (10X, 0.25 NA). The focal spot size $\left(2 \mathrm{w}_{0}\right)$ of the laser beam was $\sim 4 \mu \mathrm{m}$. Line patterns were written by moving the sample along a single axis (x-axis) with a desired separation $(50 \mu \mathrm{m})$ between the successive scan lines. A second scan directly over the machined line pattern (y-axis), under the same focusing conditions, was employed to fabricate grid patterns (Fig. 1). The same procedure was repeated for laser fluence ranging from $262 \mathrm{~mJ} / \mathrm{cm}^{2}$ to $734 \mathrm{~mJ} /$ $\mathrm{cm}^{2}$. The details of the experimental setup can be found elsewhere (George et al., 2014).

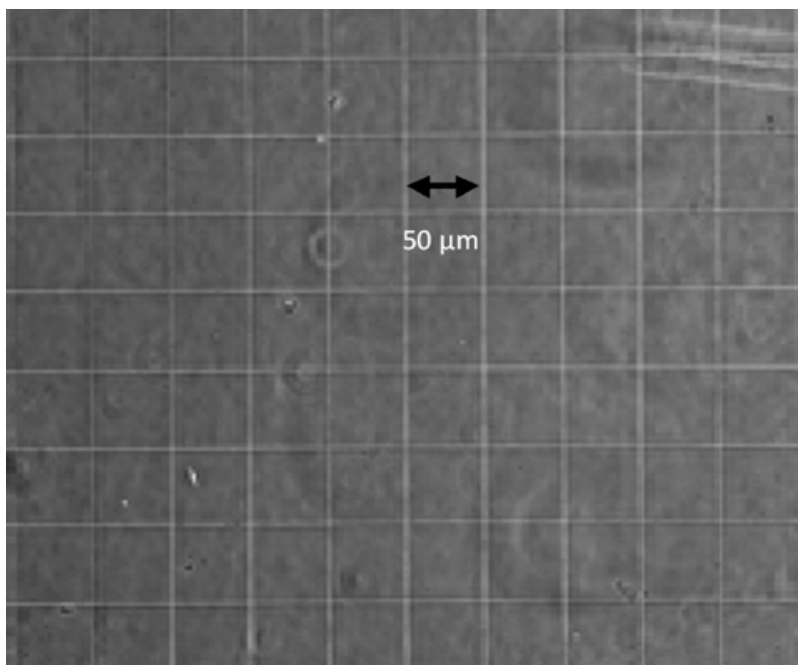

Fig. 1: Optical microscope image of grid pattern fabricated on PMMA using femtosecond laser

\section{Replication of Laser-Patterned Structures on PDMS via Soft Lithography}

The patterns fabricated in PMMA using a femtosecond laser are replicated in an intrinsically hydrophobic soft polymer, polydimethylsiloxane (PDMS) via soft lithography technique. In this technique, the two components (pre polymer and curing agent) of the PDMS are mixed thoroughly in 20:1 ratio and then desiccated for 45 minutes to remove air bubbles. The clear solution is then poured to the cleaned laser patterned PMMA substrate and heated for 90 minutes at $55^{\circ} \mathrm{C}$. Following it, the substrate and cured PDMS is allowed to cool spontaneously to room temperature and then the PDMS is peeled off from PMMA master to obtain the inverse replicated structure. The same procedure is repeated for PMMA samples patterned at highest laser fluence $\left(734 \mathrm{~mJ} / \mathrm{cm}^{2}\right)$ and corresponding contact angle value is measured on the replica surface.

\section{Contact Angle Measurements}

We quantified differences in wettability of patterned and smooth PMMA surfaces by measuring the WCA 
of a $3 \mu$ sessile droplet in directions perpendicular $\left(\theta_{\perp}\right)$ and parallel $\left(\theta_{\mid}\right)$to the grid patterns having an inter-line spacing of $50 \mu \mathrm{m}$. The droplet volume $(3 \mu \mathrm{l})$ was chosen to minimize the effect of gravity (base area<capillary length) while still providing sufficient base area to reflect any laser-induced change in our macroscopic WCA measurement. Apart from measuring the WCA for laser-patterned surfaces, the WCA over the replicated structure in PDMS is also measured.

\section{Results and Discussion}

The measured WCA for the laser-fabricated patterns under laser fluence employed here are tabulated in Table 1. The important observations are (i) hydrophobicity of PMMA is enhanced by laser patterning and it increases with laser fluence (ii) spreading of the water droplet is distinctly anisotropic. In general, the change in WCA for laser patterned polymeric surfaces is supposed to arise from either chemical modification or topography change induced by laser radiation.

We have utilized micro-Raman spectroscopy, the details of which can be found elsewhere (Zachariah et al., 2010), to check whether the laser patterning causes any chemical modification of the surface and the results are shown in Fig 2(A). From the spectral studies, it is clear that femtosecond laser patterning does not result in any chemical modification of the surface. In order to explore surface
Table 1: WCA on grid patterned surfaces fabricated at various laser fluences

\begin{tabular}{lcc}
\hline $\begin{array}{l}\text { Laser fluence } \\
\left.\text { (in } \mathrm{mJ} / \mathrm{cm}^{2}\right)\end{array}$ & $\begin{array}{c}\text { Contact angle for } 50 \mu \mathrm{m} \text { grid patterns } \\
\text { of PMMA }\end{array}$ \\
\hline 734 & $105 \pm 3^{\circ}$ & $123 \pm 3^{\circ}$ \\
629 & $106 \pm 3^{\circ}$ & $115 \pm 3^{\circ}$ \\
524 & $85 \pm 3^{\circ}$ & $106 \pm 2^{\circ}$ \\
420 & $86 \pm 2^{\circ}$ & $83 \pm 3^{\circ}$ \\
367 & $81 \pm 2^{\circ}$ & $82 \pm 3^{\circ}$ \\
Pristine & $68 \pm 3^{\circ}$ & $68 \pm 3^{\circ}$ \\
\hline
\end{tabular}

modifications on laser-patterned samples, we performed scanning electron microscopy (SEM). Typical images of laser-irradiated areas for both low and high fluence (Fig. 2(B) and (C)) reveal that the irradiated regions of the sample are porous in nature. This laser fluence dependent enhancement in porosity results in entrapment of air beneath the water droplet that acts like a cushion for the water droplet. This rationalizes the observed enhancement in WCA.

The anisotropy in wetting arises due to difference in energy barrier that a water droplet experiences perpendicular to and parallel to the direction of patterning. We replicated these PMMA grid patterns (master structure) on PDMS by following

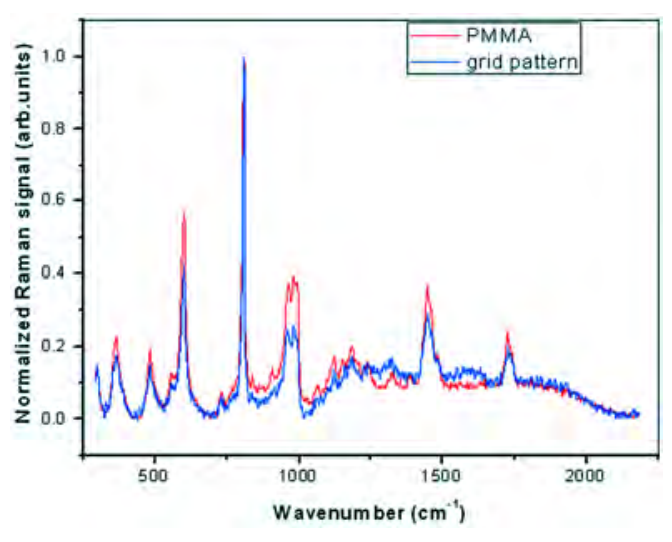

A

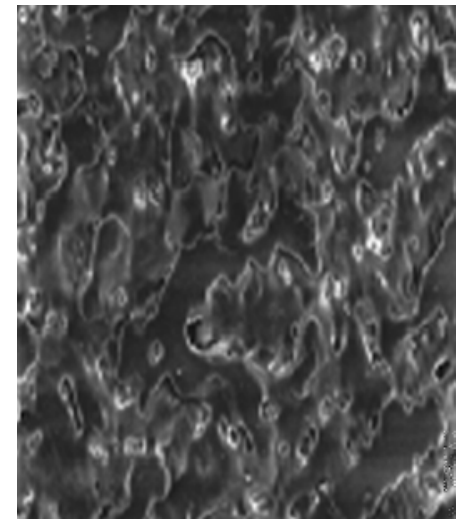

B

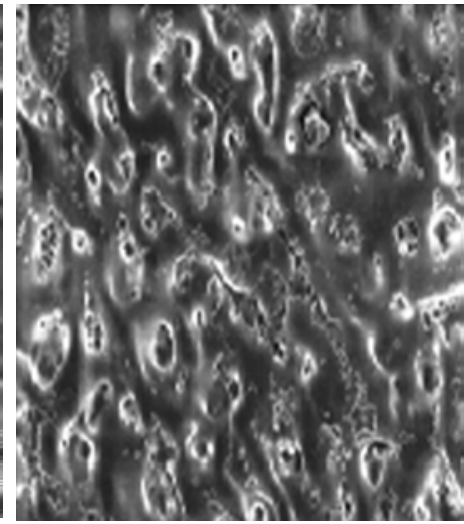

C

Fig. 2: (A) Micro-Raman spectra (B) Scanning electron microscopy images of PMMA irradiated with a fluence of $262 \mathrm{~mJ} / \mathrm{cm}^{2}$ and (C) for $734 \mathrm{~mJ} / \mathrm{cm}^{2}$. The field of view in both images is $100 \mu \mathrm{m} \times 60 \mu \mathrm{m}$ 
the above mentioned soft-lithography technique and observed a corresponding enhancement in WCA on replicated surfaces also. It is observed that the pattern replicated from PMMA structure irradiated at a laser fluence $734 \mathrm{~mJ} / \mathrm{cm}^{2}$ shows a distinctive behaviour. The dispensing of a $3 \mu$ droplet to the corresponding replicated surface in PDMS is not possible and pushing with syringe pump results in significant deformation of water droplet (as shown in Fig. 3(A)).

This clearly indicates the less adhesive force between water molecules and substrate material (superhydrophobicity). Removal of the syringe pump from the water results in rolling off of the water droplet and the movement of the droplet is limited by high surface energy difference between the pattern replicated and non-patterned regions of the PDMS (Fig. 3.(B)).

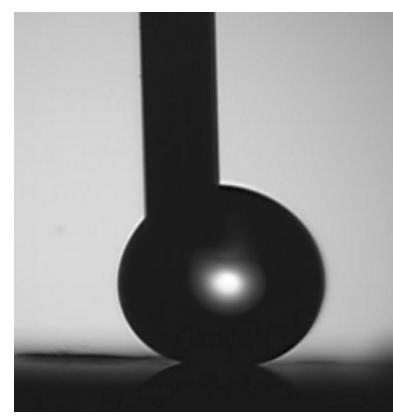

A

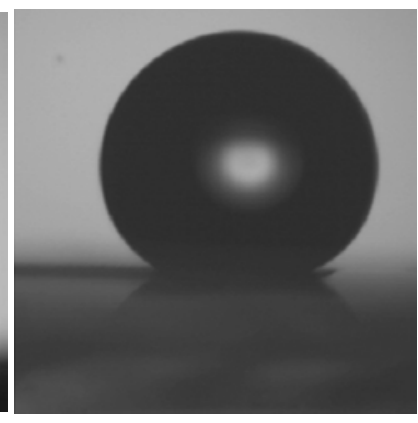

B
Fig. 3: (A) Rolling-off nature exhibited by $3 \mu$ water droplet on highest fluence $\left(734 \quad \mathrm{~mJ} / \mathrm{cm}^{2}\right)$ pattern replicated on PDMS and (B) $10 \mu \mathrm{l}$ water droplet stuck at the boundary due to energy barrier

\section{Conclusions}

In summary, femtosecond laser patterning on PMMA is found to increase the intrinsic hydrophobicity of the surface, arising from topographic modification rather than chemical modification. The patterns replicated on PDMS by using laser patterned PMMA as master structure also follow similar behaviour with a rollingoff nature for the sample prepared from the master structure created at maximum laser fluence.

\section{Acknowledgements}

This work constituted the activities of I-CUP, the Indian Cluster for Ultrafast Photonics, which was supported by the Office of the Principal Scientific Adviser to the Government of India.

\section{References}

George S D, Ladiwala U, Thomas J, Bankapur A, Chidangil S and Mathur D (2014) Deposition and alignment of cells on laser-patterned quartz Applied Surface Science 305 375381

Jeong H M, Lee W Y, Lee J H, Yang D C and Lim K S (2013) Super-hydrophobicity of PMMA and PDMS surfaces structured by femtosecond laser pulses SPIE MOEMSMEMS, International Society for Optics and Photonics, 8612

Nosonovsky M and Bhushan B (2007) Biomimetic superhydrophobic surfaces: multiscale approach Nano letters 7 2633-2637

Tang M, Shim V, Pan Z, Choo Y and Hong M (2011) Laser Ablation of Metal Substrates for Super-hydrophobic Effect $J$ Laser Micro/Nanoeng 66

Wang Z, Zheng H and Xia H (2011) Femtosecond laser-induced modification of surface wettability of PMMA for fluid separation in microchannels Microfluidics and Nanofluidics $10225-229$

Yamaguchi M, Kaneko Y and Sasaki M (2013) Fabrication of Nano-periodic Structure on Plastic Film for Water Repellent Using Femtosecond Laser Transaction on Control and Mechanical Systems 1

Zachariah E, Bankapur A, Santhosh C, Valiathan M and Mathur D (2010) Probing oxidative stress in single erythrocytes with Raman Tweezers Journal of Photochemistry and Photobiology B: Biology 100 113-116. 